Arno Gimber

\title{
W. G. Sebald y Alberto Méndez: una atrevida comparación entre la escritura postdictatorial en Alemania y España
}

Für diejenigen, die in der Zeit des Faschismus aufwuchsen, kann es kein Datum geben, von dem ab sie ihn als bewältigt erklären können.

Christa Wolf (1974)

\section{I}

La expresión española "echar en el olvido", muy defendida por los escépticos en relación con el intento de recuperación de la memoria histórica, no tiene una buena traducción al alemán. Las discusiones iniciadas a raíz de la Ley de Memoria Histórica de España, aprobada por las Cortes el 31 de octubre de 2007, y llegadas a una más exacerbada polémica por la decisión de la Audiencia Nacional de frenar las exhumaciones ordenadas por del juez Baltasar Garzón, ${ }^{1}$ tampoco tienen equivalencia exacta en otras culturas. Sería demasiado simplista querer establecer paralelismos entre el intento de recordar, a través de la literatura si es preciso, la Guerra Civil española y el régimen franquista por una parte, y la forma de tratar en textos en lengua alemana el pasado nazi por otra. Ya lo decía Aleida Assmann (2006: 70-71), en el caso español se ha producido una memoria asimétrica de la Guerra Civil, ya que hasta la muerte del dictador Francisco Franco las conmemoraciones oficiales se organizaban exclusivamente por parte de los vencedores. A una mitad de la población, a los vencidos, se les negaba llorar sus muertos en público. Estas circunstancias históricas que arrastran rencores y sufrimientos hasta hoy en día, no se corres-

1 Véase a finales de 2008 una larga serie de artículos en el diario El País, por ejemplo el de Manuel Altozano (2008) "La Audiencia suspende la apertura de fosas tras un tenso debate en la Sala Penal" o el de José Yoldi (2008) "Garzón reparte la causa del franquismo". 
ponden con ninguna realidad en la historia de los intentos de superación del pasado nazi en Alemania. ${ }^{2}$

Sin embargo, la escritura postdictatorial en países que han tenido que pasar por regímenes dictatoriales o autoritarios es comparable en muchos sentidos y a diferentes niveles y se asemeja cuando trata temas recurrentes como el trauma postdictatorial y su posible resultado en personajes múltiples o desdoblados (Gimber 2007) o cuando los autores proceden a la autocensura como reacción a una represión asumida (Peluffo 2006). Es común en la mayoría de los casos que en los primeros años tras una dictadura se produzca un silencio, una tardanza antes de poder hablar de los horrores acontecidos, fenómeno que en el caso alemán se puede estimar en unos veinte años porque hasta mediados de la década de los sesenta, quizá por la influencia de los procesos de Auschwitz ${ }^{3}$ y con la aparición del teatro documental, aparte de unas contadas excepciones, ${ }^{4}$ no hubo mención explícita del Holocausto en la literatura en lengua alemana. En el caso español, tras la muerte de Franco y la posterior Transición (que evitó indagar en los temas de la Guerra Civil y el Franquismo desviando su atención hacia el desenfreno de las diferentes movidas), podemos considerar quizá una confrontación seria con el pasado dictatorial a partir de los años noventa. Últimamente se ha producido, como es sabido, un auténtico boom $^{5}$ no solo de novelas, obras teatrales y películas sino también de estudios históricos y sociológicos sobre este tema.

$\mathrm{Si}$ en las páginas siguientes intento acercar a dos autores que se inscriben por completo en la línea de una literatura postdictatorial, no

2 Sin embargo, y también según Aleida Assmann (2006: 48), en la historia alemana se observa igualmente una incoherencia en su relación con el pasado. Existe, según la autora, un "blinder Fleck", un "punto ciego", que resulta de la dificultad "[das] ehrenhafte Verlierergedächtnis nicht vom traumatischen Tätergedächtnis trennen zu können" ("no poder separar la memoria honorable de los perdedores de la memoria traumática de los perpetradores"). Véase para su plasmación en la literatura Ulrich Krellner (2006).

3 Hay que insistir en que a diferencia del proceso de Nuremberg, de 1947, los procesos de Auschwitz fueron un asunto exclusivamente alemán.

4 Siempre son citados en este contexto de las excepciones los primeros poemas de Paul Celan, sobre todo Todesfuge.

5 En Alemania un boom de la literatura postdictatorial se produce tras la caída del muro, en 1989. Piénsese por ejemplo en los llamados Familienromane, a menudo autobiográficos, como Am Beispiel meines Bruders de Uwe Timm o Unscharfe Bilder de Ulla Hahn, ambas obras publicadas en 2003. 
pretendo, sin embargo, compararlos en su forma de escribir, sino simplemente destacar algunas coincidencias generales en su trabajo con la memoria que ojalá puedan aportar más elementos al estudio de la escritura postdictatorial en diferentes culturas con fondos históricos diferentes.

\section{II}

Los puntos comunes entre W. G. Sebald (1944-2001) y Alberto Méndez (1941-2004) no son evidentes. Aunque ambos autores pertenecen a una generación nacida en regímenes dictatoriales, el primero no lo vivió conscientemente mientras que el segundo sí hasta los 34 años (lo que significa, naturalmente, una diferencia fundamental). Ambos murieron poco después de su gran éxito como escritores: Sebald tras la publicación de su obra maestra, Austerlitz (2001), y Méndez poco después de la aparición de su único libro Los girasoles ciegos (2004, tuvo tal impacto que ya cuenta con una exitosa versión cinematográfica de José Luis Cuerda). Pero lo que más llama la atención es que tanto Los girasoles ciegos como las historias de Sebald, aparte de la novela Austerlitz los cuatro cuentos recopilados en Die Ausgewanderten (1992), son textos sobre derrotas, son textos que hablan, eso sí, de vencedores y vencidos, pero sobre todo de personas que han sufrido a raíz de guerras y de injusticias, de personas que han perdido entre tanto ruido de contienda toda una vida.

Antes de entrar en algunos detalles indicaré cuál es, siempre desde mi punto de vista, la mayor diferencia entre ambos autores, y quizá desde una perspectiva más amplia una de las más claras en los debates histórico-memorísticos en ambas culturas. La aportación de W. G. Sebald a la permanencia de ciertos recuerdos en nuestra memoria rompe con una de las tendencias más divulgadas, consistente en entender la memoria colectiva sobre todo dentro de un marco nacional. El trabajo hacia la superación del pasado es un capítulo que suele pertenecer a una nación, a su historia y a sus conflictos internos. Sebald, sin embargo, propone una visión del fenómeno, una ocupación más allá de las fronteras nacionales presentando en prácticamente todos sus libros relaciones y procesos de intercambios transnacionales y mostrándonos las secuelas del nazismo en toda Europa. Sebald propone, en resumidas cuentas, una lectura transnacional de los procesos de la memoria. 
Anteriormente ya lo hizo Hans Magnus Enzensberger en Europa in Ruinen. Augenzeugenberichte aus den Jahren 1944-1948 huyendo de la plasmación de un sufrimiento particularmente alemán e insistiendo en distintas culturas del recuerdo en toda Europa. Existen ejemplos parecidos en otras literaturas, como la japonesa cuando acude al tema de los crímenes cometidos durante la Segunda Guerra Mundial (Cornelißen/Klinkhammer/Schwentker 2003). En España no veo en la plasmación literaria postdictatorial del momento esta mirada amplia, los asuntos de la memoria colectiva se debaten más bien en casa, dentro de las fronteras de la nación. No siempre ha sido así, simplemente hay que acordarse de la literatura del exilio antifranquista que ya por su naturaleza se movía en otras delimitaciones. Max Aub sería en este contexto el más destacado ejemplo. ${ }^{6}$

Por otro lado, una de las claves para caracterizar el trabajo de la memoria, tanto en Sebald como en Méndez, se encuentra en Marcel Proust y ubico mi estudio comparativo bajo las coordenadas que él trazó en su obra monumental $A$ la recherche du temps perdu. Sebald, en varias ocasiones, fue llamado el antiproust porque al igual que el escritor francés intentaba ordenar una memoria desordenada y preconsciente, la llamada mémoire involontaire, pero siempre, y no solamente en el caso de su personaje Austerlitz, partiendo de la angustia y del trauma y no de una infancia bien cuidada como la que había vivido el protagonista de la obra de Proust. Lo que en una memoria personal se ha sumergido en el fondo desorganizado del olvido -que siempre es recuerdo latente- puede volver a la superficie bajo determinadas circunstancias con la ayuda de una clave como la famosa magdalena proustiana, que en Sebald se ha convertido, por decirlo de alguna manera, en magdalena envenenada. ${ }^{7}$ Sea como fuere, para los tres autores en cuestión, Proust, Sebald y Méndez, evocar la memoria significa poder entender las relaciones entre pasado y presente, significa ser consciente de que el pasado no es solo tiempo pretérito sino también

6 Entre los pocos ejemplos actuales comparables a Sebald destaca quizá la novela Los rojos de ultramar de Jordi Soler (2004).

7 El propio Sebald utilizó esta expresión en una entrevista para El Cultural del 2 de enero de 2002 (cf. Sánchez 2002). En Sebald la puesta en marcha de la mémoire involontaire produce igualmente sentimientos de felicidad. Sin embargo y a diferencia de Marcel Proust, enseguida son destruidos porque el encuentro con el tiempo perdido se ve envenenado por los horrores del pasado. 
actualidad que se manifiesta de manera compleja a través de los recuerdos, como bien apunta Gloria Elgueta (2000: 35). Según esta autora la memoria es construcción y elaboración social y el proceso de su re-construcción es una labor de la que se encargan nuestros autores en discursos literarios a mi parecer comparables.

En el primer tomo de $A$ la recherche du temps perdu Proust resume la recuperación de los recuerdos de la siguiente manera:

Quand d'un passé ancien rien ne subsiste, après la mort des êtres, après la destruction des choses, seules, plus frêles, mais plus vivaces, plus immatérielles, plus persistantes, plus fidèles, l'odeur et la saveur restent encore longtemps, comme des âmes, à se rappeler, à attendre, à espérer, sur la ruine de tout le reste, à porter sans fléchir, sur leur gouttelette presque impalpable, l'édifice immense du souvenir (Proust 1987: 46).

Olor y sabor son entidades poco fiables ante este edificio inmenso del pasado que difícilmente puede ser reconstruido. En los textos de Sebald los recuerdos a los que se refiere Proust no son recuperables en tanto que recuerdos individuales, ${ }^{8}$ pero parecen desembocar en una memoria cultural al igual que en la obra de Méndez cuando incluyen una dimensión colectiva. Llegar de la concepción estética de los recuerdos individuales a una concepción cultural de la memoria colectiva significa no solamente evocar edificios u objetos dignos de ser conservados en archivos y museos, sino tejer un "gewandeltes $\mathrm{Zu}-$ sammenspiel von Erinnerung und Imagination, die sich die kulturellen Archive und technischen Medien der gesellschaftlichen Kommunikation poetisch aneignet" (un "convertido juego de memoria y de imaginación que se apropia poéticamente de los archivos culturales y medios técnicos de la comunicación social", Münchberg 2005: 161; trad. de Jorge Vitón). Esta última dimensión de la apropiación literaria de otros medios de comunicación (se refiere a la inserción de fotografias, claro está) no se encuentra en los textos de Méndez.

Méndez, en su trabajo memorístico, se sitúa quizá entre Proust y Sebald, está más apegado a lo individual, pero fundamenta los recuerdos en manuscritos ficticios encontrados en archivos. Para él, como para Sebald, la restitución literaria es la reconstrucción de los currícu-

8 No se debe olvidar que Austerlitz es incapaz de reconocerse en la foto de su infancia, en la que aparece disfrazado de príncipe. En este caso la mémoire involontaire permanece muda puesto que se trata de una experiencia puramente personal. 
la de los desaparecidos. Es, sobre todo, el caso en el segundo cuento de Los girasoles ciegos (titulado Manuscrito encontrado en el olvido), que recopila unos esbozos fechados en 1940 y consultados en 1952 en los archivos generales de la Guardia Civil. Fueron encontrados en una cabaña de la montaña leonesa al lado de tres esqueletos: uno de un adulto, otro de un bebé y el tercero de una vaca. De la lectura se infiere una huida de una pareja con su niño a Francia, la muerte de la mujer y la agonía, debida a las duras condiciones del invierno, de padre e hijo escondidos en la cabaña. Termina el narrador, editor ficticio del manuscrito, con el siguiente comentario sobre el hombre muerto, Eulalio Ceballos Suárez: "Si fue él el autor de este cuaderno, lo escribió cuando tenía dieciocho años y creo que ésa no es edad para tanto sufrimiento" (Méndez 2004: 57).

\section{III}

El concepto poético tanto de Sebald como de Méndez se caracteriza por una tensión entre lo fáctico y lo ficticio, por el juego entre verificación y engaño debido a, por un lado, indicaciones exactas de tiempo y lugar y, por otro, a la imaginación. En alemán se aplica a este modo de escritura de Sebald la palabra Trübungstechnik (técnica de enturbiamiento) y Méndez nos involucra en este mecanismo cuando nos narra la historia del capitán Alegría (en el primer cuento del libro):

A partir de este documento, todos los hechos que relatamos se confunden en una amalgama de informaciones dispersas, de hechos a veces contrastados y a veces fruto de memorias neblinosas contadas por testigos que prefieren olvidar. Hemos dado crédito sin embargo a vagos recuerdos sobre frases susurradas durante ensueños angustiosos que también tienen cabida en el horror de la verdad, aunque no sean ciertos (Méndez 2004: 28).

En esta cita importan dos aspectos: primero se ve claramente (y he aquí otro punto común entre los tres autores) cómo el trabajo literario a favor de la reconstrucción de las catástrofes individuales y colectivas está vinculado con reflexiones generales sobre las prácticas de la memoria. Y segundo, queda en evidencia la incertidumbre en esta labor de la recuperación del pasado. Entre los diversos estratos narrativos, entre los diversos discursos quedan preguntas sin contestar, vacíos, sucesos sin reconstruir, silencios, quedan equívocos provocados in- 
conscientemente y falsificaciones hechas a propósito. Muy parecido, pero de forma más pesimista, se expresa Sebald al respecto:

[...] wie wenig wir festhalten können, was alles und wieviel ständig in Vergessenheit gerät, mit jedem ausgelöschten Leben, wie die Welt sich sozusagen von selber ausleert, indem die Geschichten, die an den ungezählten Orten und Gegenständen haften, welche selbst keine Fähigkeit zur Erinnerung haben, von niemandem je gehört, aufgezeichnet oder weitererzählt werden [...] (Sebald 2001: 35).

Sebald va más allá de la observación de que cualquier proceso de rememorización es un acto impreciso de reconstrucción. En la imagen tan inquietante de un mundo que se vacía a sí mismo lamenta la imposibilidad de poder recuperar el pasado. Anne Fuchs apunta que Sebald criticó en este sentido a la Alemania reunificada como un país deprimente y sin recuerdo cultural (Fuchs 2004: 247). Por eso escribe su literatura contra el olvido ya que ni siquiera la historiografía oficial -hemos aludido a la larga época de la memoria asimétrica en el caso español- garantiza el rescate del olvido de las huellas de la ignominia del pasado, sino que contribuye solamente a mantener determinados grupos en el poder gracias a construcciones de identidad o pactos de silencio.

Sea oficial o individual la memoria, los resultados de los intentos de recuperar un pasado auténtico quedan siempre borrosos. Sin embargo, la consecuencia de esta rendición nunca puede ser una negación de la representación del Holocausto (en el caso alemán) o de las atrocidades cometidas durante la Guerra Civil y después de ella (en el caso español), sino la búsqueda de representaciones adecuadas en los recursos de ficción (Stefan/Tacke 2007) ya que, a estas alturas, la autenticidad de la experiencia directa es imposible.

El autor austríaco Norbert Gstrein opina que "Geschichten vor dem Hintergrund des Dritten Reichs gibt es genug, und ich fürchte tatsächlich, es gibt schon zu viele davon" ("historias con el Tercer Reich como fondo hay suficientes y me temo que, de hecho, ya hay demasiadas" trad. de Jorge Vitón) y pregunta

9 “[...] lo poco que podemos retener, cuántas cosas y cuánto cae continuamente en el olvido, al extinguirse cada vida, cómo el mundo, por decirlo así, se vacía sí mismo, porque las historias unidas a innumerables lugares y objetos, que no tienen capacidad para recordar, no son oídas, descritas ni transmitidas por nadie [...]" (Sebald 2002a: 28). 
[...] wie man diese Geschichten noch erzählen kann, ohne der Sache bei allem guten Willen am Ende doch nur einen schlechten Dienst zu erweisen, und wenn ich Sache sage, meine ich Aufklärung, meine ich Erinnerung. Denn oft haben die Fiktionalisierungen von nicht direkt Betroffenen etwas allzu Glattes, allzu Routiniertes, sind geschenkt, wie man so sagt, weil das Ergebnis ihrer Erzählanstrengung von vornherein klar ist, manchmal nicht mehr als die Wiederholung der Tautologie, daß die Guten gut sind und die Bösen böse [...]. Es macht aus den vielen Geschichten individuellen Leids konsumierbare Geschichtchen, über die man folgenlos Rotz und Wasser heulen kann, es macht aus der Geschichte ein Spektakel, das allein deshalb nicht auf Anhieb erkennbar wird, weil es ein so schreckliches Spektakel ist (Gstrein 2003: 10-11). ${ }^{10}$

Pienso que tanto Sebald como Méndez han podido evitar los peligros evocados por Gstrein gracias a una complejidad que han introducido en sus discursos narrativos, que se ven densificados por voces múltiples. Ambos autores han jugado con, por un lado, unos recursos de la autobiografía, y por otro con referencias a varias fuentes y corroboraciones ficticias. De esta forma nacen sus obras poliperspectivisticas que de antemano huyen de cualquier peligro de simplificación como lo conocemos por ejemplo en la novela Der Vorleser de Bernhard Schlink o en películas como La vita è bella de Roberto Benigni, que carecen de sensibilidad hacia las víctimas del Holocausto.

Por cierto, en la historia del Capitán Alegría vemos además que Méndez huye de las atribuciones simplistas a un bando u otro. Alegría, nacionalista, se entrega por razones difusas a los republicanos que lo juzgan y lo condenan a muerte. Solo herido durante la ejecución consigue huir de la fosa común (aquí podría haber una referencia al protagonista de la película cuyo guión fue escrito bajo seudónimo por el propio Franco "Raza", el actor Alfredo Mayo, que también es fusilado pero sobrevive), pero cae de nuevo en manos de los suyos

10 “[...] cómo se pueden contar todavía estas historias sin, al fin y al cabo y a pesar de toda la buena voluntad, hacerle mal al asunto. Y cuando digo asunto, quiero decir esclarecimiento, quiero decir memoria. Pues, con frecuencia, las ficcionalizaciones salidas de la mano de personas no afectadas directamente resultan demasiado planas, tienen demasiada rutina, son lo que dice por gusto, porque el resultado de sus esfuerzos narrativos está claro desde el principio y en ocasiones no es más que la repetición de la tautología de que los buenos son buenos y los malos, malos [...]. De las muchas historias de sufrimiento individual estas ficcionalizaciones hacen historias consumibles con las que se puede llorar a moco tendido sin ninguna consecuencia, hacen de la historia un espectáculo que a primera vista no se reconoce como tal por el simple hecho de ser un espectáculo horrible" (Trad. de Jorge Vitón). 
(los rebeldes) que lo consideran desertor y lo encarcelan. En la cárcel se suicida para no tener que someterse a una segunda ejecución. Lo que quiero destacar, además, en esta historia es la insistencia de Méndez en el lugar fronterizo entre la vida y la muerte. "Me han juzgado pero no me han condenado. Estoy detenido en la frontera", escribe uno de los presos, Juan Senra, en la tercera historia, a su hermano (cada una de sus cartas comienza con "Sigo vivo"). Alegría, despertándose en la fosa común, se consideraba "mal enterrado entre los cuerpos desordenados de otros muertos" como si a ellos ya hubiese pertenecido. Sí, los vivos muertos y los muertos muertos forman el centro de estos textos -textos que son, al fin y al cabo, una manifestación de duelo para, así, poder salvarlos del olvido-:

Entre tantas ceremonias de muerte, tanto agotamiento, se le había escapado la vida a chorros y, preocupado solo por respirar con unos pulmones raídos por la tisis, no logró nunca saber cuál era su crimen. Solo sabía que estaban empeñados en que llegara vivo ante el pelotón de fusilamiento (Méndez 2004: 83).

$\mathrm{Y}$ en estos espacios inter, entre la vida y la muerte, se suspende el tiempo. Sebald, para conseguir esta parálisis, maniobra (entre otras) con la técnica de la mise en abyme, por ejemplo cuando mirando hacia el pasado se refleja la historia de Austerlitz en objetos, edificios o en la literatura misma (a través del juego de correspondencias entre el hipertexto Austerlitz y el hipotexto Le Colonel Chabert de Balzac).

En el tercer relato de Méndez, ya mencionado, Juan Senra aplaza su condena a muerte de día en día (como Scheherazade en Las mil y una noches) porque cuenta durante los interrogatorios diarios ante el capitán Eymar, que decidirá sobre su vida o muerte, (y ante su mujer) episodios del hijo de éstos, Miguel Eymar. Como enfermero de los republicanos Senra había conocido a Miguel Eymar, encarcelado y al final ejecutado. Los interrogatorios se convierten en recuerdos que consuelan a la madre, ávida de escuchar más y más para poder de esta forma mantener en cierto modo vivo a su hijo y a la vez, sin proponérselo explícitamente, a Senra. Como en un juego de reflejos podemos constatar que ella quiere recordar a toda costa a su hijo muerto y Senra narrar a toda costa para no morir. Para Senra, esta prolongación de su propia agonía al final resulta insoportable, confiesa sus mentiras y es condenado a muerte enseguida. 
En esta tercera historia de Los girasoles ciegos, donde constantemente se habla de la lentitud en el paso del tiempo, Méndez supera, tanto como Sebald, en lugares no seguros los límites entre los tiempos y los espacios, entre los muertos y los vivos, y así consigue denunciar en el mundo irreal los horrores cometidos como reales, y los vuelve a convertir en ficción. La mirada sinóptica, dice Sebald en Campo Santo, que recorre y traspasa los límites de la muerte, está ensombrecida, e ilumina a la vez el recuerdo de aquellos que han tenido que sufrir las mayores injusticias. Hay muchas formas de escribir sobre ello, pero solo la escritura literaria se ocupa, más allá de los hechos y de la ciencia, de un intento de restitución. En esta idea veo quizá la mayor coincidencia entre Sebald y Méndez.

¿Podemos hablar, tras este intento de comparación de las técnicas memorísticas entre ambos autores, de un heredero de Sebald en España? Hay otros muchos nombres que, para una posible comparación con Sebald, aporta la crítica literaria: Javier Marías, Roberto Bolaño, Enrique Vila-Matas (en El mal de Montano por la fusión de géneros y la yuxtaposición de texto e ilustraciones y también por la caminata entre el mundo real y el ficticio), Antonio Muñoz Molina (en Sefarad), el ya mencionado Jordi Soler o Sergio Pitol, Ricardo Piglia y Javier Cercas. ${ }^{11}$ Javier Marías desmiente (en una carta a unas de las traductoras de Sebald al castellano) su filiación y pertenencia sebaldiana, habla solo de un interés común en Thomas Browne y menciona las (pocas) fotos que él intercala en su novela Todas las almas. Sin embargo, concede a Sebald algo muy importante: pertenecer al pequeño grupo de escritores que habían conseguido en sus textos parar el tiempo, suspenderlo. Encuentra en él la visión del futuro como pasado y la del pasado como futuro, la reconciliación con los muertos y el deseo de ser uno de ellos (Gómez García 2005: 130). También en el caso de Méndez lo fugitivo, lo hemos visto, se vuelve permanente, no caduca. Este autor debería ser aceptado al igual que Sebald (nombrado "Duke of Vértigo") en el imperio imaginario llamado Redonda que se inventó Javier Marías, es decir en la comunidad de los inmortales en el paraíso de los libros (Marías 2005).

11 Se podría mencionar la reciente monografía Viaje contra espacio. Juan Goytisolo $y$ W. G. Sebald, de Jorge Carrión (2009), pero este estudio se centra en el concepto del viaje literario en ambos autores. No obstante, las alusiones al pasado y a la memoria son inevitables. 
IV

Para terminar cito de la carta que el capitán Alegría dirigió a Franco en el primer cuento de Los girasoles ciegos: "Le he escrito no para implorar su perdón, ni mostrarme arrepentido, sino para decirle que lo que yo he visto otros lo han vivido y es imposible que quede entre las azucenas olvidado" (Méndez 2004: 29). La circunstancia de este capitán franquista, mal fusilado por el enemigo y, como hemos señalado más arriba, hecho nuevamente prisionero por su propio bando tras despertar en la fosa común, vendría a confirmar lo relativo de la pertenencia a uno u otro bando. El enfoque que da Alberto Méndez al pasado es diferente al que encuentro en otras literaturas sobre el tema. Aunque insiste en la denuncia de la injusticia y de la violencia que ejercían los vencedores, los derrotados son todos, los muertos y los que siguen viviendo lamentando esas muertes. Sus textos no buscan la reconciliación, simplemente no quieren pasar página y la explicación la da el propio Méndez en la cita de Carlos Piera que antepone a su obra:

Superar exige asumir, no pasar página o echar en el olvido. En el caso de una tragedia requiere, inexcusablemente, la labor del duelo, que es del todo independiente de que haya o no reconciliación y perdón. En España no se ha cumplido con el duelo, que es, entre otras cosas, el reconocimiento público de que algo es trágico y, sobre todo, de que es irreparable (Méndez 2004: 9).

Esta reivindicación del duelo la encuentro exactamente igual en la obra de Sebald.

Quiero señalar que tras todo ello se intuye una actitud ética común entre los autores aquí tratados, un concepto de ética cercano al postulado que según Ernesto Águila Z (2005) ha formulado Adorno. Es en primer lugar la experiencia del "mal" lo que se indaga en los discursos literarios de ambos, sin que exista una idea prefijada del bien que funcione como perspectiva. No existe, como ya he señalado, la oposición entre blanco y negro. En segundo lugar, porque es una ética profundamente incrustada en una experiencia amplia, ya que implica también a la propia vivencia del lector en ella y es capaz por tanto como mimesis (en el sentido de Adorno) de producir reacciones de verdadera empatía con el dolor de los demás. He aquí una perspectiva de la escritura postdictatorial que no quiere sustentarse en una única memoria sino que se asienta en la pluralidad que establecen las diferentes cons- 
trucciones del pasado y que, de esta manera, es una propuesta seria, apropiada y adecuada para tratar, desde la ficción, la historia del fascismo en toda Europa.

\section{Bibliografía}

Águila Z, Ernesto (2005): "De Kant a Adorno: ¿un nuevo imperativo categórico?". En: Revista Perspectivas Eticas, 12, pp. 11-21 (<www.cedea.uchile.cl/ Documentos/voces\%20de\%201a\%20memoria.pdf $>$; 26.03.2009).

Altozano, Manuel (2008): "La Audiencia suspende la apertura de fosas tras un tenso debate en la Sala Penal”. En: El País, 8 de noviembre, p. 12.

Assmann, Aleida (2006): Der lange Schatten der Vergangenheit. Erinnerungskultur und Geschichtspolitik. München: Beck.

Assmann, Aleida/Frevert, Ute (1999): Geschichtsvergessenheit - Geschichtsversessenheit. Vom Umgang mit deutschen Vergangenheiten nach 1945. Stuttgart: Deutsche Verlags-Anstalt.

Carrión, Jorge (2009): Viaje contra espacio. Juan Goytisolo y W. G. Sebald. Madrid/ Frankfurt am Main: Iberoamericana/Vervuert.

Celan, Paul ([1947] 2002): Todesfuge. Aachen: Rimbaud.

Cornelißen, Christoph/Klinkhammer, Lutz/Schwentker, Wolfgang (eds.) (2003): Erinnerungskulturen: Deutschland, Italien und Japan seit 1945. Frankfurt am Main: Fischer.

Elgueta, Gloria (2000): “Secreto, verdad y memoria". En: Richard, Nelly (ed.): Politicas y estéticas de la memoria. Santiago de Chile: Cuarto Propio, pp. 33-42.

Enzensberger, Hans Magnus (1995): Europa in Ruinen. Augenzeugenberichte aus den Jahren 1944-1948. München: dtv.

Fuchs, Anne (2004): Die Schmerzspuren der Geschichte. Zur Poetik der Erinnerung in W. G. Sebalds Prosa. Köln/Wien: Böhlau.

Gimber, Arno (2007): “George-Arthur Goldschmidts Geschichte vom zerstörten Selbst”. En: Siguan, Marisa/Jané, Jordi (eds.): Erzählen müssen um zu überwinden. Literatura y supervivencia. IV Congreso de la Sociedad Goethe en España. Sitges del 22 al 24 de noviembre 2007. Barcelona: Sociedad Goethe de España, pp. 495-505.

Gómez García, Carmen (2005): “'Ruinen der Gerechtigkeit”. Zur Rezeption W. G. Sebalds in Spanien". En: Atze, Marcel/Loquai, Franz (eds.): Sebald. Lektüren. Eggingen: Isele, pp. 122-132.

Gstrein, Norbert (2003): Die Differenz. Fakten, Fiktionen und Kitsch beim Schreiben über ein historisches Thema. Wiener Rede. Frankfurt am Main: Suhrkamp.

Hahn, Ulla (2003): Unscharfe Bilder. München: DVA.

Krellner, Ulrich (2006): “Aber im Keller die Leichen/sind immer noch da'. Die Opfer-Debatte in der Literatur nach 1989”. En: Beßlich, Barbara/Grätz, Katharina/Hildebrand, Olaf (eds.): Wende des Erinnerns? Geschichtskonstruktionen in der deutschen Literatur nach 1989. Berlin: Erich Schmidt, pp. 101-114. 
Marías, Javier (2005): "Duques y Duquesas de Redonda". En: <www.javiermarias.es/ main.html> (26.03.2009).

Méndez, Alberto (2004): Los girasoles ciegos. Barcelona: Anagrama.

Münchberg, Katharina (2005): “Glückhafte Vergegenwärtigung, unheimliche Wiederkehr. Zwei Formen der Erinnerung bei Proust und W. G. Sebald”. En: Keller, Thomas (ed.): Cahier d'Études Germaniques: Transgressions/Défis/Provocations. Verstösse, Anstösse, Anstössiges. Transferts culturels franco-allemands, 1, 48 , pp. $159-172$.

Peluffo, Gabriel (2006): "Frontera_Incierta". En: <www.fronteraincierta.blogspot. com/2006/09/de-gabriel-peluffo.html> (26.03.2009).

Proust, Marcel (1987): À la recherche du temps perdu. Vol. 1. Paris: Gallimard.

Sánchez, Matilde (2002): "Entrevista a Sebald". En: El Cultural, 2-8 de enero (<www.elcultural.es/version_papel/LETRAS/3842/W_G_Sebald $>$; 03.03.2009).

Sebald, Winfried Georg (1969): Carl Sternheim. Kritiker und Opfer der Wilhelminischen Ära. Stuttgart/Berlin/Mainz: Kohlhammer.

- (1992): Die Ausgewanderten. Vier lange Erzählungen. Frankfurt am Main: Eichborn.

- (2001): Austerlitz. München/Wien: Hanser.

- (2002a): Austerlitz. Barcelona: Anagrama.

- (2002b): "Entrevista". En: El Cultural, 28.01. (<www.javiermarias.es/foro/ printthread.php?TopicID=83>; 03.03.2009).

- (2003): Campo Santo. München/Wien: Hanser.

Soler, Jordi (2004): Los rojos de ultramar. México, D.F.: Alfaguara.

Stefan, Inge/Tacke, Alexandra (eds.) (2007): NachBilder des Holocaust. Köln: Böhlau.

Timm, Uwe (2003): Am Beispiel meines Bruders. Köln: Kiepenheuer \& Witsch.

Yoldi, José (2008): “Garzón reparte la causa del franquismo”. En: El País, 19 de noviembre, p. 12. 